\title{
Euclidean Correlation Functions in a Holographic Model of QCD
}

\author{
T. Schäfer \\ Department of Physics, North Carolina State University, Raleigh, NC 27695
}

\begin{abstract}
We compute euclidean coordinate space correlation functions in a holographic model of QCD. We concentrate, in particular, on channels that are related to the $U(1)_{A}$ problem, the flavor-singlet axialvector, pseudoscalar meson, and pseudoscalar glueball (topological charge) correlator. We find that even a very simple holographic model defined on a slice of $A d S_{5}$ provides a qualitatively correct description of QCD correlation functions. We study the role of anomaly terms, and show that both euclidean positivity and low energy theorems based on the axial anomaly relation are correctly implemented. We compare the results with expectations from an instanton model of the QCD vacuum.
\end{abstract}




\section{INTRODUCTION}

Recently, much progress has been achieved in realizing the old idea that large $N$ gauge theories are related to string theory [1, 2]. The crucial development in this regard was the discovery of the correspondence between $\mathcal{N}=4$ super Yang-Mills theory and type IIB string theory on $A d S_{5} \times S_{5}$ [3]. Current effort is aimed at finding string duals of theories more closely related to QCD, in particular theories that exhibit asymptotic freedom, confinement and chiral symmetry breaking. Two avenues of research are being pursued. The top-down approach is based on the study of generalizations of the original AdS/CFT setup that incorporate fundamental fermions, chiral symmetry breaking and confinement [4, 5, 6, 7]. The bottom-up approach is founded on models of QCD that are defined on warped higher dimensional spaces and incorporate the general principles, in particular holography, of the AdS/CFT correspondence [8, 9, 10]. The aim of the bottom-up approach is to find model independent features of the holographic description, to provide guidance for the top-down approach, and to develop a geometric language for thinking about gauge theories like QCD.

Most of the published works on holographic models focus on the hadronic spectrum or on hadronic form factors. In this work we shall study euclidean correlation functions in a simple holographic model of QCD. Euclidean correlators provide a bridge between perturbation theory and the operator product expansion, which control the short distance behavior, and the mass gap, which governs the long distance behavior [11]. Phenomenological information on euclidean correlation functions comes from lattice data and from spectral functions measured in processes like $e^{+} e^{-}$annihilation into hadrons or hadronic $\tau$ decays [12, 13].

We will focus, in particular, on correlation functions related to the $U(1)_{A}$ problem. This includes the flavor-singlet axialvector, pseudoscalar meson, and pseudoscalar glueball channel. The correlators in these channels have been studied in the instanton model [14, 15, 16, 17], and there are some recent results from lattice simulations [18, 19, 20]. There are a number of qualitative questions related to the $U(1)_{A}$ sector in QCD that are still not fully understood. These include the origin of the topological susceptibility in pure gauge QCD, the microscopic mechanism that causes the susceptibility to vanish in QCD with massless fermions, and the role of instantons or other topological objects. The present work is motivated by the idea that holographic models of QCD can shed some light on these questions. 


\section{HOLOGRAPHIC MODEL}

We consider the holographic model introduced by Erlich et al. [10] and extended to the flavor singlet sector by Katz and Schwartz [21]. The model is defined by the 5-dimensional lagrangian

$$
\begin{gathered}
S=\int d^{5} x \sqrt{g}\{- \\
-\frac{1}{4 g_{5}^{2}} \operatorname{Tr}\left(F_{L}^{2}+F_{R}^{2}\right)+\operatorname{Tr}\left(|D X|^{2}+3|X|^{2}\right) \\
\left.+\frac{1}{2}|D Y|^{2}+\frac{\kappa_{0}}{2}\left(Y^{N_{f}} \operatorname{det}(X)+\text { h.c. }\right)\right\}
\end{gathered}
$$

where $X=X^{a} t^{a}$ is a scalar field, $Y$ is a complex flavor singlet scalar, $F_{\mu \nu}=\partial_{\mu} A_{\nu}-$ $\partial_{\nu} A_{\mu}-i\left[A_{\mu}, A_{\nu}\right]$ (for $\mathrm{L} / \mathrm{R}$ ) is the field strength tensor corresponding to the gauge field $A_{L, R}=A_{L, R}^{a} t^{a}$. Here, $t^{a}$ are the generators of $U(3)_{F}$. The covariant derivative is $D_{\mu} X=$ $\partial_{\mu} X-i A_{L \mu} X+i X A_{R \mu}$. The model is defined on an $A d S_{5}$ metric

$$
d s^{2}=\frac{1}{z^{2}}\left(-d z^{2}+d x^{\mu} d x_{\mu}\right)
$$

with a "hard wall" cutoff $z_{m}\left(0 \leq z \leq z_{m}\right)$. The 5-dimensional masses of the fields are determined by the correspondence between fields on $A d S_{5}$ and operators on the boundary, $m_{5}^{2}=(\Delta-p)(\Delta+p-4)$. Here, $m_{5}$ is the 5 -d mass, and $\Delta$ is the dimension of a $p$-form operator on the boundary. The scalar field $X$ corresponds to the operator $\bar{q}_{i} q_{j}$ with $\Delta=3$ and the complex scalar field $Y$ corresponds to $g^{2}(G G+i \tilde{G} G)$ with $\Delta=4$. The gauge field $A_{\mu}$ has $p=1$ and couples to the $\Delta=3$ operator $\bar{q}_{i} \gamma_{\mu} q_{j}$. The terms in the action that only involve $F_{L, R}$ and $X$ preserve the full $U(3) \times U(3)$ symmetry [10], and the last term generates the anomalous Ward identities of QCD [21].

The expectation values of $X$ and $Y$ are determined by classical solutions to the equations of motion in the presence of sources $M$ and $c$, where $M$ is the quark mass matrix, and $c$ is related to the strong coupling constant (see equ. (9) below). We have

$$
\left\langle X_{i j}\right\rangle=\sigma_{i j} z^{3}+M_{i j} z, \quad\langle Y\rangle=\Xi z^{4}+c,
$$

where $\sigma_{i j}$ and $\Xi$ correspond to the vacuum expectation values $\left\langle\bar{q}_{i} q_{j}\right\rangle$ and $\left\langle G^{2}\right\rangle$ of operators conjugate to the sources $M$ and $c$. In a more complete, top-down, model the expectation values $\sigma$ and $\Xi$ are determined dynamically [6, 7]. Alternatively, one may incorporate the dynamics of chiral symmetry breaking into a boundary action for $X$ and $Y$ [9]. Here, rather than specify these boundary terms, we shall take $\sigma$ and $\Xi$ to be free parameters of the 


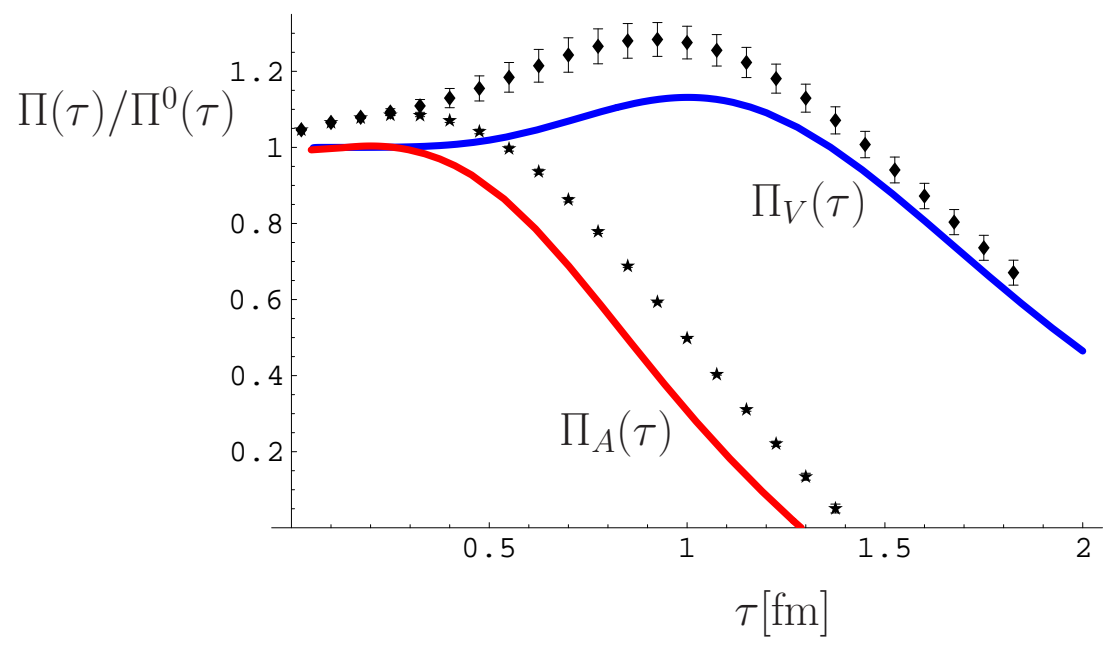

FIG. 1: Vector and axialvector current correlation functions. We show the ratio of the correlation function to the free correlator as a function of euclidean separation $\tau$. The solid curves show the result in the holographic model. The data points are taken from an analysis of Aleph data on hadronic tau decays [13].

model. In this work we are not specifically interested in flavor mixing (see [21]) and we will set $\sigma_{i j}=\delta_{i j} \sigma$ and $M_{i j}=\delta_{i j} m$.

The coupling constants $g_{5}$ and $c$ are determined by matching the short distance behavior of correlation functions to QCD. Consider the vector current correlation function

$$
\Pi_{V}\left(Q^{2}\right) \delta^{a b}\left(q_{\mu} q_{\nu}-g_{\mu \nu} q^{2}\right)=\int d^{4} x e^{i q x}\left\langle J_{\mu}^{a}(x) J_{\nu}^{b}(0)\right\rangle
$$

where $J_{\mu}^{a}=\bar{q} \gamma_{\mu} t^{a} q$ and $Q^{2}=-q^{2}$. Using the AdS/CFT dictionary we get

$$
\Pi_{V}\left(Q^{2}\right)=-\left.\frac{1}{g_{5}^{2} Q^{2}} \frac{V(q, z) \partial_{z} V(q, z)}{z}\right|_{z=\epsilon},
$$

where $V(q, z)$ is the bulk-to-boundary propagator, $V_{\mu}(q, z)=V(q, z) V_{\mu}^{0}(z)$, and $V_{\mu}^{0}(q)$ is the boundary value of the field. The propagator has to satisfy the equation of motion. In the $V_{z}(x, z)=0$ gauge the linearized equation of motion for the gauge field is

$$
\partial_{z}\left(\frac{1}{z} \partial_{z} V_{\mu}^{a}(q, z)\right)+\frac{q^{2}}{z} V_{\mu}^{a}(q, z)=0 .
$$

A solution on the complete $A d S_{5}$ space with $V(q, z \rightarrow 0)=1$ is given by $V(q, z)=$ $-\frac{\pi}{2}(q z) Y_{1}(q z)$ and

$$
\Pi_{\mathrm{V}}\left(Q^{2}\right)=-\frac{1}{2 g_{5}^{2}} \log \left(Q^{2}\right) .
$$


This result can be compared to the perturbative one-loop contribution $\Pi_{V}\left(Q^{2}\right)=$ $-N_{c} /\left(24 \pi^{2}\right) \cdot \log \left(Q^{2}\right)$. This leads to the matching condition

$$
g_{5}^{2}=\frac{12 \pi^{2}}{N_{c}} .
$$

We observe that $g_{5}^{2} \sim 1 / N_{c}$ which shows that vector mesons at large $N_{c}$ are weakly coupled, as expected from general large $N_{c}$ arguments. The constant $c$ is fixed by matching the flavorsinglet axialvector correlation function and using the axial anomaly relation. The result is [21]

$$
c=\sqrt{2 N_{f}} \frac{\alpha_{s}}{2 \pi^{2}},
$$

and we shall follow [21] and allow $\alpha_{s}$ to run as a function of $z$ according to the one-loop beta function. Having fixed the coupling constants we can now compute the correlation functions. For this purpose we shall employ the representation of the Green function in terms of eigenfunctions of the five-dimensional Sturm-Liouville problem. In the vector channel

$$
\Pi_{V}\left(Q^{2}\right)=\sum_{k} \frac{f_{\rho, k}^{2}}{m_{\rho, k}^{2}\left(Q^{2}+m_{\rho, k}^{2}\right)}
$$

where $q^{2}=m_{\rho, k}^{2}$ is an eigenvalue of equ. (6) subject to the boundary conditions $V(0)=0$ and $V^{\prime}\left(z_{m}\right)=0$. The eigenfunctions are normalized according to

$$
\int d z \frac{1}{z} V_{k}(z) V_{l}(z)=\delta_{k l}
$$

and the decay constants $f_{\rho, k}$ are given by

$$
f_{\rho, k}=\left.\frac{1}{g_{5}} \frac{V^{\prime}(z)}{z}\right|_{z=\epsilon} .
$$

The eigenmode representation (10) also determines the spectral function and the euclidean correlator. The spectral function is a sum of delta-functions, and the euclidean correlation function is

$$
\Pi_{V}(\tau)=\sum_{k} f_{\rho, k}^{2} D\left(m_{\rho, k}, \tau\right), \quad D(m, \tau)=\frac{m}{4 \pi^{2} \tau} K_{1}(m \tau),
$$

where $D(m, \tau)$ is the euclidean propagator of a scalar particle with mass $m$. In the vector channel the spectrum can be obtained almost in closed form. The eigenvalues $m_{\rho, k}$ are determined by the zeros of the Bessel function of the first kind and order zero, $J_{0}\left(m_{\rho, k} z_{m}\right)=$ 


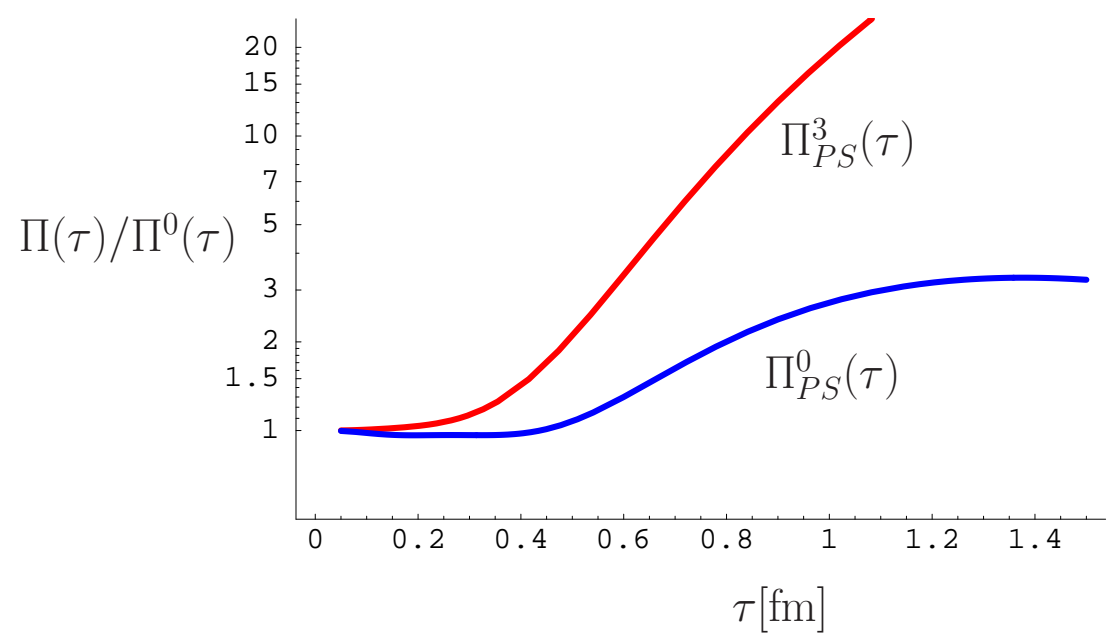

FIG. 2: Pseudoscalar octet and singlet current correlation functions. The correlation functions are normalized to the correlator in the free theory.

0. Using the location of the first zero and the physical mass of the rho meson we can set the scale, $z_{m}=0.62 \mathrm{fm}$. The coupling constant of the k'th resonance is given by

$$
f_{\rho, k}=\frac{\sqrt{2}}{g_{5}} \frac{m_{\rho, k}}{z_{m} J_{1}\left(m_{\rho, k} z_{m}\right)} .
$$

The euclidean correlation function is shown in Fig. 1. We plot the ratio of the euclidean correlator $\Pi_{V}(\tau)$ over the correlator in the non-interacting theory, $\Pi_{V}^{0}(\tau)=N_{c} /\left(3 \pi^{4} \tau^{6}\right)$. Because of asymptotic freedom this ratio approaches one as $\tau \rightarrow 0$. The result can be compared to the experimental results compiled in [13]. The agreement is quite good, but at short distances perturbative logarithms and power corrections are missing. The short distance behavior can be improved by adding extra terms to the action given in equ. (11), but this is beyond the scope of the present work. The agreement with experimental data is comparable to resonance saturation models constrained by Weinberg sum rules and other QCD inputs [22, 23]. This is not surprising, as equ. (10) is equivalent to the resonance saturation ansatz, equ. (8) is the statement of local duality, and Weinberg sum rules are realized as a consequence of chiral symmetry. 


\section{PSEUDOSCALAR AND AXIALVECTOR CORRELATION FUNCTIONS}

The equations of motion for the transverse components of the axialvector gauge field is

$$
\partial_{z}\left(\frac{1}{z} \partial_{z} A_{\mu}^{a}\right)+\frac{q^{2}}{z} A_{\mu}^{a}-\frac{g_{5}^{2} v^{2}}{z^{3}} A_{\mu}^{a}=0
$$

where we have introduced the notation $v=m z+\sigma z^{3}$ for the vacuum expectation value of the scalar field. This equation differs from the equation for the vector field by an extra contribution due to (explicit and spontaneous) chiral symmetry breaking. The longitudinal component of the axialvector current mixes with the pseudoscalar current, and pseudoscalar fluctuations have be to taken into account. We define octet $\eta^{a}(a=1, \ldots, 8)$ and singlet $\eta^{0}, a$ fields by

$$
X_{i j}=\left\langle X_{i j}\right\rangle \exp \left(i \eta^{a} t^{a}\right), \quad Y=\langle Y\rangle \exp (i a)
$$

We also define the longitudinal component of the axialvector field $\partial_{\mu} \varphi^{a}=A_{\mu}^{a}-A_{\mu \perp}^{a}$ (in $A_{z}^{a}=0$ gauge). We first consider the flavor non-singlet sector. The equations of motion for the longitudinal axialvector $\varphi^{a}$ and the pseudoscalar field $\pi^{a}$ are

$$
\begin{aligned}
\partial_{z}\left(\frac{1}{z} \partial_{z} \varphi^{a}\right)+\frac{g_{5}^{2} v^{2}}{z^{3}}\left(\pi^{a}-\varphi^{a}\right) & =0 . \\
-q^{2} \partial_{z} \varphi^{a}+\frac{g_{5}^{2} v^{2}}{z^{2}} \partial_{z} \pi^{a} & =0 .
\end{aligned}
$$

The solutions are normalized according to

$$
\int d z \frac{v^{2}}{z^{3}} \pi_{k}^{a}\left(\pi_{l}^{a}-\varphi_{l}^{a}\right)=\delta_{k l}
$$

and the pion decay constant is given by

$$
f_{\pi, k}=\left.\frac{1}{g_{5}^{2}} \frac{\partial_{z} \varphi_{k}^{a}}{z}\right|_{\epsilon} .
$$

We consider $N_{c}=3$ and set $m=2.23 \mathrm{MeV}$ and $\sigma=(323 \mathrm{MeV})^{3}$ in order to reproduce the experimental values of $m_{\pi}$ and $f_{\pi}$ for the lowest pion excitation. The trace of the axialvector correlation function, $\Pi_{A} \equiv \Pi_{A \mu}^{\mu}$, is shown in Fig. 1. The splitting between the vector and axialvector correlators is related to the difference in mass and coupling between the vector and axialvector mesons, and to the pion contribution. The latter dominates at large distance and causes $\Pi_{A}$ to become negative. In the model considered here chiral symmetry is restored high in the spectrum $\left(m_{\rho, k}^{2} \rightarrow m_{a_{1}, k}^{2}\right.$ as $\left.k \rightarrow \infty\right)$ and the vector and axialvector correlators are very nearly degenerate for $\tau<0.5 \mathrm{fm}$. 
In the singlet sector there is also mixing with the pseudoscalar glueball field $a$. The equations of motion are

$$
\begin{aligned}
\partial_{z}\left(\frac{1}{z} \partial_{z} \varphi^{0}\right)-g_{5}^{2} \frac{v^{2}}{z^{3}}\left(\varphi^{0}-\eta^{0}\right)-g_{5}^{2} \frac{c^{2}}{z^{3}}\left(\varphi^{0}-a\right) & =0 \\
\partial_{z}\left(\frac{c^{2}}{z^{3}} \partial_{z} a\right)+q^{2} \frac{c^{2}}{z^{3}}\left(a-\varphi^{0}\right)+\kappa \frac{v^{N_{f}}}{z^{5}}\left(\eta^{0}-a\right) & =0 \\
q^{2} z^{2} \partial_{z} \varphi^{0}-g_{5}^{2} v^{2} \partial_{z} \eta^{0}-g_{5}^{2} c^{2} \partial_{z} a & =0,
\end{aligned}
$$

where we have defined $\kappa=c^{N_{f}} \kappa_{0}$. The normalization condition is

$$
\int d z\left[\frac{v^{2}}{z^{3}} \eta_{k}^{0}\left(\eta_{l}^{0}-\varphi_{l}^{0}\right)+\frac{c^{2}}{z^{3}} a_{k}\left(a_{l}-\varphi_{l}^{0}\right)\right]=\delta_{k l} .
$$

For a normalized eigenmode the coupling constants to the axialvector, pseudoscalar quarkanti-quark, and pseudoscalar glueball current are given by

$$
f_{\eta^{\prime}, k}=\left.\frac{1}{g_{5}^{2}} \frac{\partial_{z} \varphi_{k}^{0}}{z}\right|_{\epsilon}, \quad \lambda_{\eta^{\prime}, k}=\left.m \frac{\partial_{z} \eta_{k}^{0}}{z}\right|_{\epsilon}, \quad h_{\eta^{\prime}, k}=\left.\frac{c^{2}}{\sqrt{2 N_{f}}} \frac{\partial_{z} a_{k}}{z^{3}}\right|_{\epsilon} .
$$

In Fig. 2 we show the pseudoscalar correlation functions

$$
\Pi_{P}\left(Q^{2}\right) \delta^{a b}=\int d^{4} x e^{i q x}\left\langle\bar{q} t^{a} \gamma_{5} q(x) \bar{q} t^{b} \gamma_{5} q(0)\right\rangle
$$

in the flavor singlet and non-singlet sector. In the non-singlet sector the correlation function is dominated by the light pion excitation. In the singlet sector the would-be Goldstone boson receives an extra contribution to its mass from the anomaly term. We have set $\kappa=20$ and

find $m_{\eta^{\prime}}=650 \mathrm{MeV}$. (For $\kappa \gg 1$ the mass of the $\eta^{\prime}$ becomes weakly dependent on the value of $\kappa$. We also note that the experimental result for the $\eta^{\prime}$ mass, $m_{\eta^{\prime}}=957 \mathrm{MeV}$, can be reproduced if the strange quark mass is taken into account.) Like chiral symmetry, the axial $U(1)_{A}$ symmetry is effectively restored in the highly excited part of the spectrum. For $k \gg 1$ the equations of motion for $\eta^{0}, \varphi^{0}$ and $a$ effectively decouple, and the solutions to Eqs. (21-23) alternate between solutions to the equations of motion in the non-singlet and the glueball sector of the theory.

\section{TOPOLOGICAL CHARGE CORRELATOR}

The pseudoscalar glueball correlation function is

$$
\Pi_{P}\left(Q^{2}\right)=-\int d^{4} x e^{i q x}\left\langle\frac{\alpha_{s}}{8 \pi} G \tilde{G}(x) \frac{\alpha_{s}}{8 \pi} G \tilde{G}(0)\right\rangle .
$$




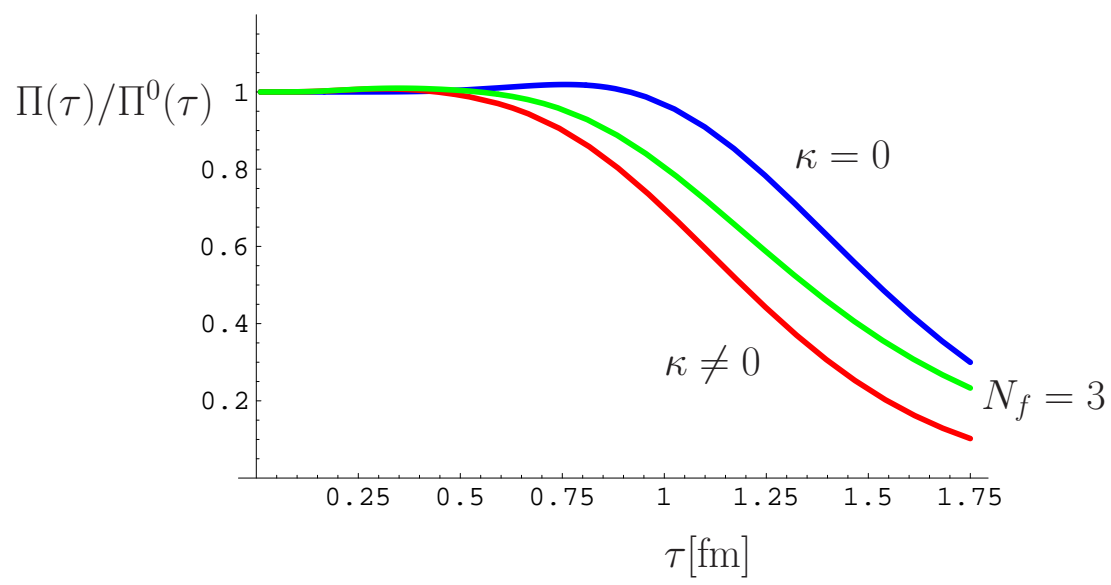

FIG. 3: Pseudoscalar glueball correlation functions. The correlation functions are normalized to free field behavior. The curve labeled $\kappa=0$ corresponds to the pure gauge theory without anomaly term, whereas the curve labeled $\kappa \neq 0$ includes the effects of an anomaly term. The curve labeled $N_{f}=3$ shows the result in full QCD, including mixing with the $\eta^{\prime}$.

Note that $\alpha_{s} /(8 \pi) \cdot G \tilde{G}$ is the topological charge density. In order to study the topological charge correlator in the holographic model we begin with the equations of motion in the pure gauge sector of the theory. We have

$$
\partial_{z}\left(\frac{c^{2}}{z^{3}} \partial_{z} a\right)+q^{2} \frac{c^{2}}{z^{3}} a-\frac{\bar{\kappa}}{z^{5}} a=0
$$

where $\bar{\kappa}=\kappa v^{N_{f}}$. The solutions of this equation are particularly simple in the limit $c=$ const and $\bar{\kappa} \rightarrow 0$. In this case the eigenvalues in the pseudoscalar glueball channel are given by the solutions of $J_{1}\left(m_{G, k} z_{m}\right)=0$ and the coupling constants are

$$
h_{G, k}=\frac{c}{2 \sqrt{N_{f}}} \frac{m_{G, k}^{2}}{z_{m} J_{2}\left(m_{G, k} z_{m}\right)} .
$$

The groundstate in the pseudoscalar glueball channel is heavier than the ground state rho meson by a factor $\sim 1.5$. The glueball correlation function is shown in Fig. 3, The shape of the correlation function is similar to the vector meson correlator, but since the mass scale is larger the correlator is smaller. The topological susceptibility is defined as the integral of the pseudoscalar glueball correlation function

$$
\chi_{\text {top }}=\lim _{V \rightarrow \infty} \frac{\left\langle Q_{\text {top }}^{2}\right\rangle}{V}=-\int d^{4} x \Pi_{P}(x)
$$




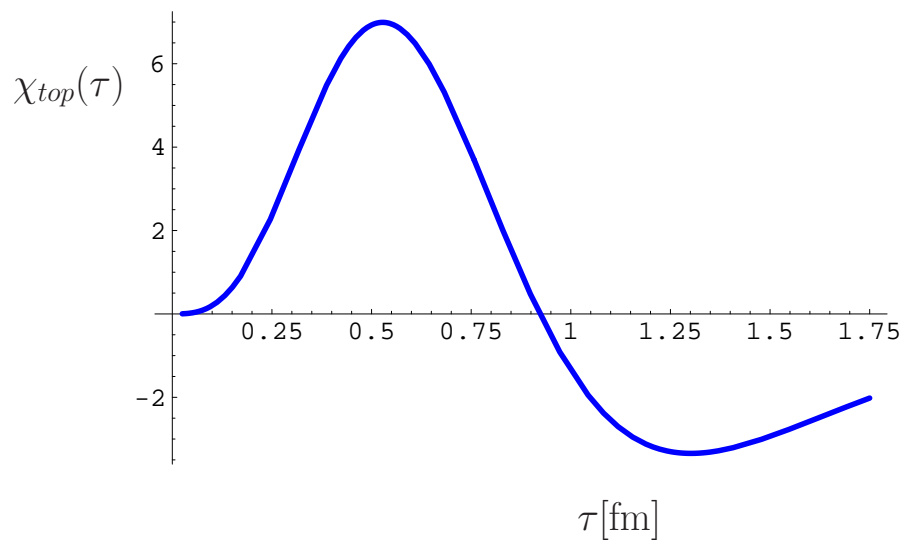

FIG. 4: Integrand for topological susceptibility in pure gauge QCD without an anomaly term. The integrand changes sign at $z \sim 1 \mathrm{fm}$, and the topological susceptibility vanishes.

where $Q_{\text {top }}$ is the topological charge and $V$ is the volume. This integral diverges at short distance, and care has to be taken in defining the subtraction scheme. In the present case it is sufficient to subtract the contribution from the free gluon bubble, $\Pi_{P}^{0}=6 \alpha_{s} /\left(\pi^{6} x^{8}\right)$. This corresponds to subtracting the correlator in the infinite (non-cutoff) $A d S_{5}$ space. The integrand $2 \pi^{2} x^{2}\left(\Pi_{P}(x)-\Pi_{P}^{0}(x)\right)$ is shown in Fig. 4. We observe that the integrand changes sign, and we find that the topological susceptibility vanishes. This result, as well as the subtraction scheme, can be checked by computing the topological susceptibility directly from the solution of the equation of motion (28) for $q^{2}=0$. The only solution that satisfies the boundary conditions $a(0)=1$ and $\partial_{z} a\left(z_{m}\right)=0$ is $a(z)=1$, and the topological susceptibility

$$
\chi_{\text {top }}=-\left.\frac{c^{2}}{2 N_{f}} \frac{\partial_{z} a}{z^{3}}\right|_{\epsilon} .
$$

vanishes. This result does not depend on the simplifying assumption $c=$ const. It does, however, depend on the choice $\kappa=0$. The correlation function for $\kappa \neq 0$ is also shown in Fig. 3. The anomalous term suppresses the correlator and leads to a non-vanishing (and positive) topological susceptibility. In the simplest model, defined by equ. (28), the topological susceptibility has a non-perturbative ultra-violet divergence. This can be seen by treating the anomaly term as a small correction. In this limit the topological charge correlator is [21]

$$
\Pi_{P}(Q)=-\frac{1}{2 N_{f}} \int_{0}^{z_{m}} \frac{d z}{z^{5}} \bar{\kappa}\left[\frac{1}{2}(Q z)^{2} K_{2}(Q z)\right]^{2},
$$




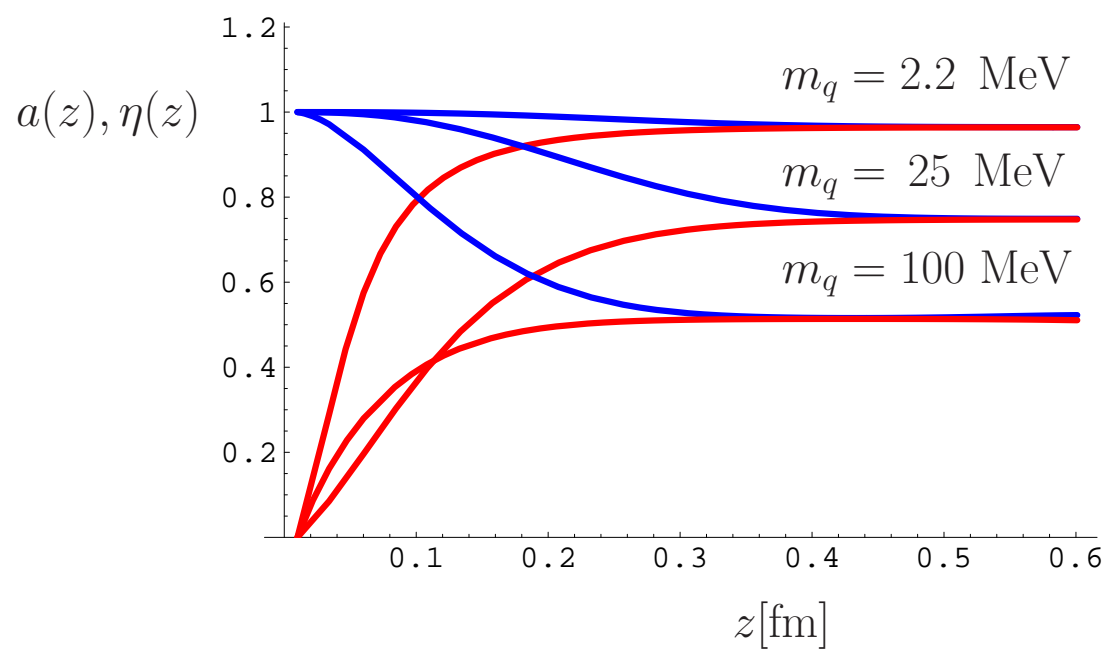

FIG. 5: Five dimensional $q^{2}=0$ modes in the pseudoscalar sector. We show solutions of the equations of motion for $a(z)$ (blue, solutions start at $a(0)=1)$ and $\eta^{0}(z)$ (red, satisfy $\eta^{0}(0)=0$ ) for different values of the quark mass $m$. The topological susceptibility is related to the negative of the curvature of $a(z)$ near $z=0$.

where the expression in the square brackets is the bulk-to-boundary propagator of the $a$ field in the infinite $A d S$ geometry (for $\bar{\kappa}=0$ ). For $Q^{2}=0$ the integrand is singular near $z=0$ unless $\bar{\kappa}(z)$ vanishes faster than $z^{4}$ as $z \rightarrow 0$. This problem can be understood by comparing equ. (32) with the expectation from an instanton model, see appendix A. In terms of instantons the integral $d z / z^{5}$ arises from the integral over the size of the instanton, and the power $z^{5}$ is fixed by classical scale invariance. The Bessel function corresponds to the Fourier transform of the topological charge density, and the order of the Bessel function is determined by classical scale invariance and the dimension of the operator $G \tilde{G}$. The instanton contribution in QCD contains an extra factor $\exp \left(-8 \pi^{2} / g^{2}(z)\right)$, where $8 \pi^{2} / g^{2}$ is the instanton action, and $g(z)$ is the running coupling constant. Asymptotic freedom implies that this term scales as $z^{b}$ (for $z \rightarrow 0$ ), where $b$ is the first coefficient of the beta function. As a consequence, the integral is well behaved for small $z$, but diverges at large scale sizes. In the holographic model the infrared problem is cured by the hard wall cutoff, but the simplest version of the model has an ultraviolet divergence instead.

The problem can be addressed by considering a more complicated functional form for $\bar{\kappa}(z)$. Here, we proceed directly to the unquenched theory, which has a finite topological susceptibility. The topological charge correlator in the theory with three flavors is shown in 
Fig. (3). There is an extra contribution which is dominated by mixing with the lowest $\eta^{\prime}$ resonance. This term increases the topological charge correlator at long distance, and tends to cancel the topological susceptibility generated in the pure gauge theory. The topological susceptibility can be determined most accurately by solving the equations of motion for $Q^{2}=0$,

$$
\begin{aligned}
\partial_{z}\left(\frac{c^{2}}{z^{3}} \partial_{z} a\right)+\kappa \frac{v^{N_{f}}}{z^{5}}\left(\eta^{0}-a\right) & =0, \\
v^{2} \partial_{z} \eta^{0}+c^{2} \partial_{z} a & =0,
\end{aligned}
$$

subject to the boundary conditions $a(0)=1, \eta^{0}(0)=0, \partial_{z} a\left(z_{m}\right)=\partial_{z} \eta^{0}\left(z_{m}\right)=0$. Solutions to equs. (33-34) for different values of the quark masses are shown in Fig. 5 , We observe that the topological susceptibility scales with the quark mass, as expected from low energy

theorems based on the axial anomaly [24, 25]. For $m=2.2 \mathrm{MeV}$ we obtain $\chi_{\text {top }}=(70 \mathrm{MeV})^{3}$, in agreement with the expected value $\chi_{\text {top }}=m \sigma / N_{f}$.

\section{SUMMARY}

We have studied euclidean coordinate space correlation functions in a holographic model of QCD. The correlation functions in the flavor non-singlet vector and axialvector channel agree well (within $\sim 20 \%$ ) with experimental data from hadronic tau decays. The holographic model does not describe perturbative logarithms and power corrections. In principle this can be addressed by modifying the $A d S_{5}$ geometry, or by adding higher dimensional terms in the five-dimensional action.

In the pure gauge sector, without an anomaly term, the model generates a non-trivial pseudoscalar glueball spectrum but the topological susceptibility is zero. The anomaly term gives a negative (repulsive) contribution to the pseudoscalar glueball correlator and leads to a non-zero topological susceptibility. In the full theory an extra attractive contribution arises from mixing between the pseudoscalar glueball and the pseudoscalar meson $\left(\eta^{\prime}\right)$ field. These two terms cancel as the quark mass goes to zero, and the topological susceptibility vanishes. The attractive term is longer ranged than the repulsive one, so the vanishing of $\chi_{\text {top }}$ can be viewed as being due to topological charge screening. It also interesting to compare the results to expectations from the instanton model. The anomaly contribution to 
both the topological charge and the pseudoscalar meson correlator agree with the structure of the dilute instanton gas result.

In this work we studied a very schematic model based on a slice of $A d S_{5}$. Clearly, it is of interest to study correlation functions in "top-down" models. A very detailed study of anomalous correlators in the $\mathcal{N}=4$ theory was carried out by Dorey et al. [26] Some attempts to study topology and the $\eta^{\prime}$ meson in extensions of the simplest AdS/CFT setup can be found in [27, 28, 29, 30, 31, 32]. It is also important to further clarify the role of instantons in the large $N_{c}$ limit. Witten argued that instanton effects are suppressed in the weak coupling limit of a large $N$ field theory [33]. In QCD the situation is not clear, because the theory is classically conformal (it has instantons of all sizes), and in the large $N_{c}$ limit only small instantons are suppressed. We have previously argued that the instanton size distribution in the large $N_{c}$ limit might be a delta function [34]. In the language of holographic QCD this corresponds to an anomaly term $\kappa(z)$ which is sharply peaked at some critical distance $z^{*}$ in the fifth dimension.

Acknowledgments: Part of this work was completed at the Newton Institute for Mathematical Sciences during the workshop on Strong Fields, Integrability and Strings. I would like to thank the Institute for their hospitality. This work is supported by a grant from the United States Department of Energy, \#DE-FG02-03ER41260. I would also like to thank M. Schwartz for useful correspondence.

\section{APPENDIX A: CORRELATION FUNCTIONS IN THE FIELD OF AN INSTAN-} TON

In this appendix we collect a few results for the single instanton contribution to hadronic correlation functions. The instanton contribution to the pseudoscalar glueball correlation function is [14, 15, 16]

$$
\Pi_{P}(Q)=-2 \int \frac{d \rho}{\rho^{5}} d(\rho)\left[\frac{1}{2} Q^{2} \rho^{2} K_{2}(\rho Q)\right]^{2},
$$

where the Bessel function $K_{2}(\rho Q)$ arises from the Fourier transform of the topological charge density of an instanton. The factor 2 arises from adding the contribution of instantons and anti-instantons. The instanton size distribution is given by [35]

$$
d(\rho)=C_{N_{c}}\left(\frac{8 \pi^{2}}{g^{2}}\right)^{2 N_{c}} \exp \left(-\frac{8 \pi^{2}}{g(\rho)^{2}}\right) \prod_{f} m_{f}^{*} \rho
$$




$$
\begin{aligned}
m_{f}^{*} \rho & =m \rho-\frac{4 \pi^{2}}{3}\langle\bar{q} q\rangle \rho^{3} \\
C_{N_{c}} & =\frac{0.466 \exp \left(-1.679 N_{c}\right) 1.34^{N_{f}}}{\left(N_{c}-1\right) !\left(N_{c}-2\right) !}
\end{aligned}
$$

where $g(\rho)$ is the one-loop running coupling constant

$$
\frac{8 \pi^{2}}{g^{2}(\rho)}=-b \log (\rho \Lambda), \quad b=\frac{11}{3} N_{c}-\frac{2}{3} N_{f} .
$$

The factor $1 / \rho^{5}$ is related to classical scale invariance and matches the determinant of the metric tensor on $A d S_{5}$. We also note that the factor $\left(m^{*} \rho\right)^{N_{f}}$ matches the factor $v^{N_{f}}=$ $\left(m z+\sigma z^{3}\right)^{N_{f}}$ in equ. (33). The remaining terms in $d(\rho)$ are related to fluctuations around the classical instanton solution and have no obvious counterpart in the holographic model.

The instanton contribution to the flavor non-singlet pseudoscalar meson correlation function is [36]

$$
\Pi_{\pi}(Q)=2 \int \frac{d \rho}{\rho^{5}} d(\rho) \frac{1}{m^{2}}\left[Q \rho K_{1}(\rho Q)\right]^{2} .
$$

This result includes only the contribution of the fermion zero mode in the quark propagator. The instanton contribution to the flavor singlet correlator is the same up to an overall sign. The Bessel function $K_{1}(\rho Q)$ is the Fourier transform of the fermion zero mode. The order is related to the dimension of the Fermion field, and matches the bulk-to-boundary propagator for the pseudoscalar field on $A d S_{5}$.

The instanton contribution to the vector current correlation function is [37]

$$
\Pi_{\mu \nu}(Q)=\left(q_{\mu} q_{\nu}-\delta_{\mu \nu} q^{2}\right) \frac{4}{3} \int \frac{d \rho}{\rho^{5}} d(\rho)\left\{\frac{1}{Q^{4}}-\frac{3 \rho^{2}}{Q^{2}} \int_{0}^{1} d x K_{2}\left(2 \rho Q /\left(1-x^{2}\right)^{1 / 2}\right)\right\} .
$$

The first term is a pure power correction which corresponds to the $\left\langle g^{2} G^{2}\right\rangle$ and $m\langle\bar{q} q\rangle$ terms in the OPE. These terms are not present in the holographic model. The second term, which is due to non-zero modes of the fermion propagator in the instanton field, also has no counterpart in the model considered here.

[1] G. 't Hooft, Nucl. Phys. B 72, 461 (1974).

[2] A. A. Migdal, Annals Phys. 109, 365 (1977).

[3] J. M. Maldacena, Adv. Theor. Math. Phys. 2, 231 (1998) [Int. J. Theor. Phys. 38, 1113 (1999)] arXiv:hep-th/9711200. 
[4] J. Polchinski and M. J. Strassler, arXiv:hep-th/0003136.

[5] A. Karch and E. Katz, JHEP 0206, 043 (2002) arXiv:hep-th/0205236.

[6] J. Babington, J. Erdmenger, N. J. Evans, Z. Guralnik and I. Kirsch, Phys. Rev. D 69, 066007 (2004) arXiv:hep-th/0306018.

[7] T. Sakai and S. Sugimoto, Prog. Theor. Phys. 113, 843 (2005) arXiv:hep-th/0412141.

[8] A. A. Migdal, Annals Phys. 110, 46 (1978).

[9] L. Da Rold and A. Pomarol, Nucl. Phys. B 721, 79 (2005) arXiv:hep-ph/0501218.

[10] J. Erlich, E. Katz, D. T. Son and M. A. Stephanov, Phys. Rev. Lett. 95, 261602 (2005) arXiv:hep-ph/0501128.

[11] M. A. Shifman, A. I. Vainshtein and V. I. Zakharov, Nucl. Phys. B 147, 385 (1979); Ibid. 448.

[12] E. V. Shuryak, Rev. Mod. Phys. 65, 1 (1993).

[13] T. Schäfer and E. V. Shuryak, Phys. Rev. Lett. 86, 3973 (2001) arXiv:hep-ph/0010116.

[14] B. V. Geshkenbein and B. L. Ioffe, Nucl. Phys. B 166, 340 (1980).

[15] V. A. Novikov, M. A. Shifman, A. I. Vainshtein and V. I. Zakharov, Phys. Lett. B 86, 347 (1979) [JETP Lett. 29, 594.1979].

[16] T. Schäfer and E. V. Shuryak, Phys. Rev. Lett. 75, 1707 (1995) arXiv:hep-ph/9410372.

[17] T. Schäfer and E. V. Shuryak, Rev. Mod. Phys. 70, 323 (1998) arXiv:hep-ph/9610451.

[18] S. O. Bilson-Thompson, D. B. Leinweber and A. G. Williams, Annals Phys. 304, 1 (2003) arXiv:hep-lat/0203008.

[19] I. Horvath et al., Phys. Lett. B 617, 49 (2005) arXiv:hep-lat/0504005.

[20] P. J. Moran and D. B. Leinweber, arXiv:0710.2380 [hep-lat].

[21] E. Katz and M. D. Schwartz, JHEP 0708, 077 (2007) [arXiv:0705.0534 [hep-ph]].

[22] M. A. Shifman, arXiv:hep-ph/0009131.

[23] O. Cata, M. Golterman and S. Peris, JHEP 0508, 076 (2005) arXiv:hep-ph/0506004.

[24] E. Witten, Nucl. Phys. B 156, 269 (1979).

[25] G. Veneziano, Nucl. Phys. B 159, 213 (1979).

[26] N. Dorey, T. J. Hollowood, V. V. Khoze, M. P. Mattis and S. Vandoren, Nucl. Phys. B 552, 88 (1999) arXiv:hep-th/9901128.

[27] E. Witten, Phys. Rev. Lett. 81, 2862 (1998) arXiv:hep-th/9807109].

[28] A. Hashimoto and Y. Oz, Nucl. Phys. B 548, 167 (1999) arXiv:hep-th/9809106.

[29] C. T. Hill and P. Ramond, Nucl. Phys. B 596, 243 (2001) arXiv:hep-th/0007221. 
[30] M. Kruczenski, D. Mateos, R. C. Myers and D. J. Winters, JHEP 0405, 041 (2004) arXiv:hep-th/0311270].

[31] J. L. F. Barbon, C. Hoyos, D. Mateos and R. C. Myers, JHEP 0410, 029 (2004) arXiv:hep-th/0404260].

[32] A. Armoni, JHEP 0406, 019 (2004) arXiv:hep-th/0404248.

[33] E. Witten, Nucl. Phys. B 149, 285 (1979).

[34] T. Schäfer, Phys. Rev. D 66, 076009 (2002) arXiv:hep-ph/0206062.

[35] G. 't Hooft, Phys. Rev. D 14, 3432 (1976) [Erratum-ibid. D 18, 2199 (1978)].

[36] E. V. Shuryak, Nucl. Phys. B 214, 237 (1983).

[37] M. S. Dubovikov and A. V. Smilga, Nucl. Phys. B 185, 109 (1981). 